\title{
LA AUSENCIA DE CORRESPONSABILIDAD, FRENO PARA EL DESARROLLO DE LA CARRERA LABORAL FEMENINA EN LA ACADEMIA
}

\author{
THE LACK OF JOINT RESPONSIBILITY, BRAKE FOR THE \\ DEVELOPMENT OF THE FEMALE CAREER IN ACADEMIA
}

\author{
Estrella MONTES LÓPEZ \\ Universidad de Salamanca \\ estrellamontes@usal.es \\ orcid.org/0000-0002-8226-7504
}

\section{Resumen}

Este artículo presenta parte de los resultados de una investigación sobre el desarrollo de la carrera profesional en la Universidad abordada desde la perspectiva de género. Concretamente, se centra en la relación entre el trabajo académico y la vida familiar a través del análisis de entrevistas en profundidad realizadas a profesorado universitario en una Universidad pública española. Los resultados de investigación muestran, por un lado, la difícil conciliación de la vida laboral y familiar de quienes trabajan en la Academia y, por otro, los discursos de las personas entrevistadas en torno a la ausencia de corresponsabilidad en los hogares como una de las causas de la desigual posición de la mujer en la Universidad.

Palabras clave: Universidad, carrera académica, género, conciliación, corresponsabilidad.

\begin{abstract}
This article presents part of the results of a research on the development of the career in the university. Specifically, it focuses on the relationship between academic work and family life through the analysis of in-depth interviews conducted to academics in a Spanish public university. On the one hand, the results of the research show the difficult work-life balance in academia. On the other hand, they show the discourse of the people interviewed about the lack of joint responsibility in couples as one of the causes of the unequal position of women in university.
\end{abstract}


Keywords: university, academic career, gender, working-life balance, joint responsibility 


\section{INTRODUCCIÓN}

Este artículo es parte de una investigación más amplia sobre el desarrollo de la carrera profesional en la Universidad, abordada desde la perspectiva de género, en la que se desarrollaron entrevistas en profundidad a profesorado universitario para conocer las explicaciones que este aporta en relación a la desigual posición de la mujer. Actualmente, y pese a que las mujeres han representado alrededor del $40 \%$ del profesorado universitario en los últimos años, tienen una desigual presencia en las diferentes categorías laborales. Así, a medida que aumenta la posición en la escalera académica, disminuye la presencia de mujer. La media nacional de mujeres catedráticas de Universidad en las universidades públicas españolas en el curso 2015-2016 era tan solo del 20,88\% (según los últimos datos actualizados del Ministerio de Educación, Cultura y Deporte 2017). De igual modo, la mujer está desigualmente representada en los principales cargos de gestión académica, esto es, como rectoras, como decanas y como directoras de departamento (Tomàs-Folch y Guillamón).

De manera general, las explicaciones aportadas por los participantes en la investigación pueden agruparse en tres grandes bloques: aquellas que defienden que las causas de tal desigualdad recaen en las características de las propias mujeres; las que la explican como resultado de factores relacionados con la propia organización, como la estructura de la carrera, los procesos de reclutamiento y selección en la Universidad o la importancia de las relaciones personales; y las que se centran en poner el foco de atención en un nivel mucho más amplio, el nivel institucional o social, indicando que el problema está en la estructura social y en cómo esta afecta al trabajo. Estas últimas explicaciones son las que se exponen en este artículo. Así, los objetivos que se persiguen son, en primer lugar, visibilizar los problemas de conciliación de la esfera familiar con la laboral que surgen en la Universidad, especialmente en el caso de las mujeres, ya que son ellas las continúan asumiendo principalmente las responsabilidades familiares; y en segundo término, mostrar cómo esta situación de ausencia de corresponsabilidad en los hogares acarrea efectos en la carrera laboral de las mujeres, limitando su desarrollo. 
Este artículo se divide en cinco partes. En primer lugar se expone, con cierta perspectiva histórica, el diferente empleo del tiempo que realizan hombres y mujeres. En segundo término se ahonda en la literatura sobre la división sexual del trabajo doméstico para conocer las consecuencias que esta tiene en las mujeres. En un tercer momento se explica brevemente la metodología de investigación, para posteriormente exponer los resultados de investigación, estructurados en tres grandes bloques, según se refieran a la conciliación de los tiempos de trabajo en general, a la difícil conciliación familiar y laboral en la carrera académica o a la ausencia de corresponsabilidad como causa de la desigual promoción de la mujer en la Universidad. Por último, se exponen las conclusiones y reflexiones que estos hallazgos arrojan.

\section{EL DIFERENTE USO DEL TIEMPO DE HOMBRES Y MUJERES}

El tiempo es una de las dimensiones clave de la organización de la vida humana, y su importancia yace de que «frente a la inmensidad del tiempo posible, el tiempo humano es parcial, acotado» (Durán 1986, 13). Sin embargo, no solo es limitado y escaso, y con ello, de importante valor, sino que además es construido. La construcción personal del tiempo varía en función de la posición que ocupa cada sujeto en la estructura familiar, y este empleo desigual del tiempo genera distintas consecuencias en las diferentes esferas de la vida, esto es, a nivel personal, familiar y laboral. Como señala Durán (2010), el tiempo que dedicamos a dormir nos iguala, «homogeneiza las edades, las clases sociales y los géneros» (34), sin embargo, el tiempo que pasamos despiertos, es tiempo de diferencia.

Durán (1986) revelaba en su obra, hace más de treinta años, la situación de la mujer de la época, adscrita a la producción doméstica a través del cumplimiento de las normas sociales imperantes. Así, la mayor parte del tiempo de las mujeres no era intercambiado en el mercado laboral (como si lo era en el caso de los hombres), sino empleado en la producción de servicios domésticos para la unidad familiar. Su proyecto de vida era un proyecto colectivo que incluía a la familia y acababa en la vejez. Al contrario, la trayectoria laboral de los hombres se concebía como un proyecto a largo plazo, individualizado y ascendente hasta la jubilación.

Treinta y un años después de ese estudio, y tras los grandes cambios sociales acaecidos en España, cabría esperar que los usos del tiempo entre hombres y mujeres fueran muy diferentes en la actualidad. Sin embargo, los múltiples estudios sobre esta temática, y a pesar de la evolución en los usos del tiempo, han ido poniendo en evidencia el mantenimiento de diferencias en el empleo del tiempo entre hombres y mujeres (Ramos; Álvaro 1996a 1996b; Instituto 
de la Mujer 2007; Prieto, Ramos y Callejo; Durán 2010; Domínguez). Estudios recientes muestran que se ha producido una reducción de la desigualdad, pero que siguen existiendo grandes diferencias por sexo: las mujeres siguen empleando más del doble de tiempo que los hombres al trabajo reproductivo, mientras estos trabajaban fuera del hogar una hora y cuarto más y dedican alrededor de 50 minutos más al día que ellas a actividades de ocio. Así, y aunque la diferencia entre la carga global de trabajo (la suma del trabajo productivo y reproductivo) masculino y femenino ha disminuido en relación al pasado, la mujer continúa trabajando tres cuartos de hora más al día que el hombre (Instituto de la Mujer 2013), y disfrutando de casi una hora menos de ocio que él (Conde-Ruiz y Marra). Durán (2010) nos invita a calcular lo que supone el tiempo que la mujer trabaja más que el hombre al día a lo largo de la vida. El resultado equivale a varios años de trabajo que las mujeres realizan a mayores frente a los hombres. Pese a que esto puede considerarse una abultada especulación, bien merece nuestra reflexión, ya que una hora de tiempo, un día, no supone a priori mucho tiempo, sin embargo, su progresiva acumulación es una cuestión diferente.

\section{LA DIVISIÓN DEL TRABAJO DOMÉSTICO Y SUS CONSECUENCIAS PARA LAS MUJERES}

Carrasco considera que el principal conflicto del siglo XXI es el cuidado de la familia y del hogar, debido a que la masiva incorporación de la mujer al mercado laboral no ha ido de la mano de una similar vinculación de los hombres al ámbito reproductivo (Beck y Beck-Gernsheim; Carrasco; García; Tobío 2012; González y Jurado). Actualmente, sigue faltando democracia interna en la familia (Anguita, Alario y Ortiz) y sigue sorprendiendo que pese a que la mujer se haya incorporado al ámbito de lo público, su presencia en el espacio privado sea casi la misma que era en el pasado (Tobío 2012; González y Jurado).

El trabajo de cuidado sigue recayendo principalmente sobre la mujer, que es a quien tradicionalmente se ha socializado para desempeñarlo. Estudios como el desarrollado por el Instituto de la Juventud (2008), reflejan que en el seno de muchas familias se sigue favoreciendo la asunción de determinadas tareas del hogar, consideradas tradicionalmente como femeninas, por parte de niñas y adolescentes, en comparación con sus hermanos varones. El hecho de que el trabajo de cuidado siga recayendo sobre las mujeres genera que cuando estas se incorporan al mercado de trabajo, se produzca una superposición de sus nuevos y antiguos roles laborales, recayendo sobre ellas la responsabilidad de hacerlos compatibles. Así, la conciliación del trabajo productivo y reproductivo se hace a costa de las propias mujeres, asumiendo una mucho 
mayor carga de trabajo, un «doble turno» (Hochschild; Domínguez) o una «doble jornada laboral» (Tobío 2001; Donoso, Figuera y Rodríguez; González y Jurado). Esta «jornada múltiple», como también se refieren a ella Donoso, Figuera y Rodríguez (2011), implica no solo la suma del resto del jornadas, sino «una superposición de estas, con todas las problemáticas que conlleva» (190).

A nivel social, cuando hablamos de «trabajo», solemos entender el intercambio mercantil que se produce entre un empleador y un trabajador, retribuyendo el primero al segundo por su actividad laboral. La no remuneración del trabajo realizado en el seno del hogar genera, por un lado, que este permanezca invisible y, por otro, que se supedite al trabajo asalariado. Fruto de ello, este trabajo no solo no se valora, sino que no se reconoce la doble dedicación que realizan las mujeres (Varella; Tobío 2012) y el conflicto que viven como resultado de ella (Carrasco). Sin embargo, esta doble jornada laboral acarrea efectos sobre la salud (física, psicológica y social), sobre la relación con el trabajo y sobre la imagen social de las mujeres (Barberá y Ramos; Prieto, Ramos y Callejo).

La desigual situación de la que parte la mujer en la sociedad y en el mercado de trabajo en general, también tiene efectos cuando esta desarrolla su trabajo en la Academia. Las profesoras de Universidad, como hacen otras mujeres en otros espacios de trabajo, reproducen los principios y roles sociales aprendidos en la vida académica (Anguita, Alario y Ortiz), lo que genera formas diferenciadas de participación por sexo, pero también su exclusión de algunas etapas del proceso científico. Así, por ejemplo, las mujeres tienen una menor presencia en las redes informales, realizan menos investigaciones en coautoría, o son menos citadas que sus compañeros varones (Reskin; García de León y García de Cortázar). Concretamente, en el siguiente apartado se abordan las dificultades de conciliación de la vida personal y el trabajo en la Universidad, y el efecto que la ausencia de corresponsabilidad en los hogares tiene sobre la carrera académica de las mujeres.

\section{METODOLOGÍA}

Esta investigación, como se ha señalado, forma parte de un estudio más amplio sobre el desarrollo de la carrera académica y género en la Universidad. En él se recogieron y analizaron los testimonios de profesorado de una Universidad pública española para conocer, desde el punto de vista de los propios implicados, cómo se produce el desarrollo de la carrera profesional en la Universidad y las posibles diferencias por sexo. Así, se realizaron 43 entrevistas en profundidad a profesorado universitario teniendo en cuenta cuatro variables para diversificar los discursos de los participantes: su sexo (aplicándose tanto a hombres como a mujeres); las cargas familiares (tener o no tener hijos a cargo); 
la rama de conocimiento en la que desarrolla su trabajo, diferenciando de manera genérica entre Letras (áreas de Artes, Humanidades, Ciencias Sociales y Jurídicas) y Ciencias (áreas de Ciencias, Ciencias de la Salud, Arquitectura e Ingeniería); y el puesto de trabajo dentro de la jerarquía académica universitaria (siendo también entrevistadas personas que estaban ocupando cargos de gestión a nivel departamental, de facultad o en el equipo de gobierno).

El contenido de la entrevista versó acerca de la elección de la carrera académica, del desarrollo de la misma (atendiendo especialmente a las motivaciones, los momentos más relevantes y más gratificantes, y a las barreras y pasos más difíciles), de la relación entre la vida personal y la carrera profesional, y de las posibles diferencias en el trabajo y trayectorias laborales de hombres y mujeres en la Universidad.

Todas las entrevistas en profundidad fueron grabadas y transcritas fielmente. La duración media de las entrevistas fue de una hora y dieron lugar a más de 700 páginas de texto de análisis. El trabajo de sistematización, codificación, exploración, comparación y análisis de las entrevistas fue realizado con el apoyo del programa informático ATLAS.ti y se tomaron como unidades de análisis tanto palabras relevantes, como frases, párrafos o fragmentos del texto. A continuación se exponen los resultados de investigación, acompañados por citas textuales extraídas de los testimonios de las participantes con el objetivo de favorecer su comprensión.

\section{CONCILIACIÓN Y CORRESPONSABILIDAD EN LA UNIVERSIDAD}

En la exposición de resultados se abordan, en primer lugar, los diferentes modelos de relación que existen entre el trabajo productivo y reproductivo; en segundo término, las dificultades de conciliación de la vida familiar y laboral en el caso del trabajo en la Academia; y en último lugar, cómo parte de las personas entrevistadas entienden la ausencia de corresponsabilidad en los hogares como causa de la desigual promoción de la mujer en la Universidad.

\subsection{La conciliación de los tiempos de trabajo}

Como sostienen Prieto y colaboradores, «ningún tiempo social es inteligible en sí mismo, sino sólo en el marco de sus relaciones con los otros» (Prieto, Ramos y Callejo, 355), pero esto no significa que todos los tiempos tengan el mismo peso para las personas, al contrario, los tiempos de trabajo productivo y reproductivo se imponen sobre el resto de tiempos (Acker; Prieto, Ramos y Callejo). Además, aunque se realice una diferenciación entre uno y otro, ambos tienen características comunes: a) suponen esfuerzo y con ello desgaste 
físico-mental; b) requieren ser planificados y organizados; c) necesitan de un aprendizaje específico para su realización y d) conllevan un resultado social a través de la interacción social (Murillo; Lozares, López y Martí).

Lozares, López y Martí describen los diferentes modelos de relación que se producen entre estos dos tipos de trabajo. Uno de los modelos está caracterizado por una doble centralidad segmentada y desigualmente distribuida por género, donde las actividades, tiempos y espacios de trabajo productivo y reproductivo está dividida, correspondiendo el primero a los hombres y el segundo a las mujeres. Este es el modelo estricto de división sexual del trabajo. Otro de ellos, contrapuesto al anterior, estaría constituido por una centralidad negociada entre el hombre y la mujer, donde la carga total de trabajo, y las responsabilidades, tareas y tiempo que lleva anexas, estaría equilibradamente distribuida entre ambos.

Entre estos dos modelos polarizados existe otro intermedio, donde se han producido avances hacia la igualdad, pero aún continua siendo una asignatura pendiente, y en el que pueden diferenciarse dos situaciones. En el primer caso, el hombre tiene centralidad productiva pero no reproductiva, y la mujer tiene una débil centralidad productiva (trabaja fuera del hogar pero su responsabilidad, conocimientos y experiencia son débiles) y una alta centralidad reproductiva. En esta situación, la mujer tiene muchas dificultades para conseguir una centralidad productiva elevada, más allá de la mera realización del trabajo, lo que incidirá perpetuando el desarrollo del trabajo reproductivo sobre ella. En el segundo caso, ambos sujetos tienen la máxima centralidad productiva, pero el hombre tiene una centralidad insignificante en el trabajo reproductivo (realiza mínimas actividades, sin asumir la responsabilidad de la organización y con pocos conocimientos y experiencia), en comparación con la mujer, que tiene también la máxima centralidad reproductiva. Por tanto, esta asume una doble centralidad, que implica doble presencia, doble dedicación, y necesidad de equilibrar ambas esferas. Tanto el trabajo productivo como el reproductivo son «una parte central de su identidad» (Lozares, López y Martí 174), pero su asunción le causará tensiones de compatibilización de tiempos y espacios.

Un reciente estudio coordinado por González y Jurado sobre la corresponsabilidad en parejas jóvenes muestra que existen tres modelos de distribución del trabajo en las parejas, los cuales presentan muchas similitudes con los propuestos por Lozares y sus colaboradores. El primero de ellos, que responde al modelo tradicional, está compuesto por una madre que realiza la mayor parte del trabajo de cuidados mientras el padre cuida solo de manera ocasional debido a su centralidad en el trabajo remunerado. El segundo modelo está en 
transición entre los otros dos. En él, el varón ayuda en las tareas de cuidado pero sin asumir toda la responsabilidad sobre ellas, mientras la mujer asume una alta y doble carga de trabajo, y con ello, la dificultad de conciliar la vida familiar con la laboral. Esto a menudo la obliga a tener que anteponer el trabajo de cuidados al desarrollo profesional. Por último, el tercero es un modelo nuevo en el que ambos padres desarrollan trabajos fuera del hogar a la vez que son corresponsables en el cuidado de los hijos y en la toma de decisiones sobre él. Este estudio también muestra que a pesar de los valores de igualdad en la sociedad, el reparto desigual del trabajo sigue siendo elevado. En él se entrevista a parejas heterosexuales que comparten las tareas del hogar, antes y después de tener su primer hijo. Los autores eligieron el parto como punto de inflexión basándose en la literatura que afirma que la desigualdad en el reparto de tareas aumenta a partir de la llegada de los hijos. Así, y a pesar de que partían de una situación de igualdad, observan que no todas las parejas logran mantener esa distribución, lo que obliga a las mujeres a asumir la doble carga o a realizar renuncias laborales.

Respecto a las entrevistas realizadas para esta investigación, no cabe duda de que las mujeres entrevistadas revelan una alta centralidad productiva, y en el caso de las que tienen hijos, también tienen una alta centralidad reproductiva, aunque el grado de participación de sus parejas varíe desde una mínima a una amplia participación en el trabajo doméstico y de cuidados. Así, las mujeres entrevistadas con cargas familiares se sitúan en el modelo intermedio, asumiendo en la mayor parte de los casos el peso y dificultades de la conciliación laboral. Tan solo una mujer muestra una situación familiar bastante cercana a la corresponsabilidad en el hogar. Por su parte, los profesores varones entrevistados revelan modelos familiares muy dispares, evidenciando ejemplos de los tres tipos. Algunos varones tienen esposas que no trabajan fuera del hogar, y con ello, se ocupan del trabajo reproductivo, eximiéndolos de él. Otros participan en el hogar ayudando a sus parejas en el trabajo doméstico, aunque son ellas las que asumen mayor responsabilidad y dedicación a él. Ningún hombre con hijos manifiesta, ni se desgrana de su testimonio, ser corresponsable, ni siquiera en los casos de los varones más jóvenes.

\subsection{La difícil conciliación familiar y laboral en la carrera académica}

De manera casi unánime, las personas entrevistadas consideran que la conciliación personal y familiar con la carrera académica es muy complicada, principalmente por la necesidad de dedicar tiempo «privado» (el que queda fuera de la jornada laboral legalmente establecida) al trabajo para poder avanzar y promocionar en esta carrera. Usualmente, las personas entrevistadas usan 
metáforas como «carrera de fondo», «carrera de obstáculos» o «contrarreloj» para referirse a la carrera académica, indicando que hay que trabajar mucho y de manera continuada para superar las diferentes etapas con sus correspondientes requerimientos, que a pesar del duro trabajo se tarda mucho tiempo en acumular méritos, y, a su vez, que hay que tratar de conseguirlos en el menor tiempo posible para cumplir los plazos establecidos y competir con los demás. De este modo hacen hincapié en que es una carrera que no permite descanso. Al contrario, se necesita alargar la jornada laboral, trabajar los fines de semana y hasta en vacaciones. Señalan que la jornada laboral diaria apenas da para impartir clases, realizar tutorías, evaluar a los alumnos y cumplir con las múltiples burocracias, quedando muy poco tiempo para la investigación. Así, se requiere utilizar el tiempo que debería ser de ocio o descanso para continuar desarrollándola. Además, esta situación se ve agravada en el caso de quienes ocupan cargos de gestión. Teniendo presente todo ello, gran parte del profesorado entrevistado considera que otras muchas profesiones son más fáciles de conciliar con la vida privada que la Academia:

Hay profesiones y profesiones. A lo mejor una profesión mucho más ordenada, pues un bancario, que tiene sus horas, tiene sus tal, pues es mucho más fácil convivir con este tipo de personas ¿no? Donde sabes que tienes un horario y cuando terminas el horario de trabajo tú puedes disponer de todo tu tiempo, y tu cabeza la vacías porque te dedicas a hacer otras cosas (E.23. Hombre. Contratado doctor. Con hijos. Letras).

Algunas personas entrevistadas llegan a cuestionarse cómo el actual diseño de la carrera académica afecta a la estructura familiar del profesorado universitario. Por ejemplo, el siguiente entrevistado sostiene que las exigencias de dedicación a la carrera académica, que reducen el tiempo disponible haciéndolo muy escaso y dificultando la conciliación, afectan a la vida social del profesorado, llegando a generar rupturas en las relaciones de pareja, que se reduzca el número de hijos a tener (frente al que les gustaría) o incluso que la carrera profesional en la Universidad se considere incompatible con tener hijos:

Me gustaría hacer una encuesta, ¿cuántos profesores de la universidad española, en estos momentos, de menos de 45 años están casados? ¿Cuántos, en qué perfiles de edad? Segunda pregunta ¿cuántos de ellos tienen hijos y cuántos hijos tienen? [...] Yo creo que la situación en la universidad española es muy compleja y ha entrado en un círculo vicioso, un círculo vicioso motivado yo creo por el incrementar la calidad en los indicadores y por reducir los costes salariales (E.24. Hombre. Contratado doctor. Con hijos. Letras).

Hay quien incluso llega a considerar que la carrera universitaria es incompatible con la vida personal: «Claro, yo, la carrera universitaria, en la carrera 
del profesor, es absolutamente incompatible con la vida personal, pero vamos absolutamente» (E.19. Cargo de gestión. Titular de Universidad. Sin hijos. Letras). En unos casos u otros, y dentro de la difícil conciliación de la vida laboral y privada, las personas entrevistadas habitualmente destacan que hacer compatibles ambas esferas es mucho más fácil para aquellos que no tienen cargas familiares, ya que pueden disponer con mayor libertad de su tiempo para ponerlo a disposición de la carrera profesional. Esto ha sido evidenciado por diversos estudios: la carga de trabajo en el ámbito doméstico y de cuidados afecta al ámbito productivo (Gutek y Larwood; Tharenou; Martínez-Pérez y Osca). La siguiente entrevistada habla de su propio caso: «Ser Catedrática requiere dedicar muchísimo tiempo, bueno pues a cuestiones de investigación y también de docencia, y bueno, si una persona es soltera como yo, pues no tiene hijos, y esto hace que yo pueda dedicar más tiempo» (E. 11. Mujer. catedrática de Universidad. Sin hijos. Letras).

La mayor parte de las alusiones a las cargas familiares en esta investigación hacen referencia al cuidado de los hijos, sin embargo, en ocasiones también se destaca la dificultad de conciliación de quienes tienen personas mayores a su cargo. Así, la siguiente entrevistada, por ejemplo, cuenta cómo tuvo que renunciar al cargo gestor que estaba ocupando cuando sus padres enfermaron (era la única hija de una familia numerosa, donde los hijos varones rehusaron de hacerse cargo de sus padres): «Ha habido un momento en el que yo he tenido que cortar eso porque tenía mi madre muy enferma, y mi padre tiene Alzheimer. Pues claro, una situación familiar que era incompatible con mis otras dedicaciones, así que tuve, realmente lo tuve que dejar» (E.19. Cargo de gestión. Titular de Universidad. Sin hijos. Letras). Por su parte, la siguiente entrevistada no solo menciona los problemas de conciliación por cuidado de personas mayores, sino que a su vez, reconoce que esto afectó menos a su carrera de lo que otras cargas familiares pueden afectar a otras personas, porque ella asumió tal responsabilidad cuando ya había alcanzado la categoría de catedrática de Universidad (la entrevistada 20 plantea un situación similar, como se expone más adelante). De este modo, manifiesta también la difícil conciliación y el efecto que tiene en la carrera académica la asunción de responsabilidades familiares cuando la persona se encuentra en los primeros escalones. Particularmente, se refiere a las mujeres y a la maternidad:

Eso sí que condiciona un poco, el tener que conciliar la vida familiar con la vida profesional. A mí me pilla en un momento que tengo que conciliar mi vida familiar con mi vida profesional, en un momento en que digamos, yo ya, desde el punto de vista académica, yo ya soy catedrática. Digamos, he conseguido... Pero, claro, conseguir una, culminar una etapa, eso, no significa que después no tengas que, que seguir al pie del cañón. Entonces, sí que me he 
dado cuenta de la necesidad de esa conciliación merma un poco tus expectativas $i$ no? Por eso puedo entender que cuando esa necesidad se produce en las primeras etapas de tu vida académica, algunas etapas se tengan que retrasar (E.12. Catedrática de Universidad. Sin hijos. Ciencias).

El profesorado entrevistado no solo coincide en indicar que la conciliación de la vida privada y laboral es más complicada para aquellos que tienen cargas familiares, sino que además reconoce que es más duro para las mujeres, debido a que son ellas las que común y principalmente asumen las responsabilidades domésticas y de cuidado de los miembros de la familia. Además de la ausencia de corresponsabilidad en los hogares, la difícil conciliación de ambas esferas se hace aún más complicada por la inexistente conciencia universitaria de tener en cuenta la vida familiar, que hace que los tiempos de trabajo y familia choquen, cuando podría ser fácilmente evitable. Así lo refleja la entrevistada 14 , que expone que la fijación de reuniones en horarios que chocan con los de la familia es una práctica habitual, lo que genera que las mujeres se vean obligadas a abandonar estos encuentros antes de su finalización o a llamar para que alguien solucione la desatención que estar en dicha reunión ocasiona en la familia. Considera, además, que esto no sucede en el caso de los varones. En primer lugar, esta participante ejemplifica una situación de hace años, cuando era más joven:

Solían convocarnos por la tarde, a las seis, o una cosa así, no recuerdo porqué, pero sé que era la hora a la que nos solíamos reunir. Entonces hasta las ocho aguantábamos allí todo el mundo, pero a partir de las ocho pues ya empezaban las obligaciones familiares a llamar ¿no? Y sistemáticamente, um, nos íbamos, o se iban las mujeres. Los hombres no tenían prisa para irse a casa isabes? (E.14. Mujer. Cargo de gestión. Titular de Universidad. Con hijos. Ciencias)

En segundo lugar, cuenta una situación sucedida varios días antes, en la que son convocados por un hombre a una reunión a la hora de comer, que llegadas las tres de la tarde esta aún no ha finalizado, y que las únicas que muestran preocupación por esta situación, porque afecta al ámbito familiar, son las mujeres:

En la vida personal las mujeres seguimos teniendo otra carga, absolutamente distinta. [...] Era la hora de comer, y al Vicerrector de turno que nos había citado no le importaba tenernos a la tres de la tarde esperando, y los compañeros que estaban del grupo, no les importaba. Pero las que éramos mujeres, tenías que llamar a casa «Oye que no sé qué», porque la que tenía organizada la comida eras tú. Entonces, no veo diferencias aquí, pero sí que en nuestra vida personal las mujeres seguimos teniendo un papel diferente y lo notas en todas las compañeras, absolutamente (E.14. Mujer. Cargo de gestión. Titular de Universidad. Con hijos. Ciencias) 
Muchas de las personas entrevistadas consideran que la mujer sigue asumiendo en mayor medida que los hombres las responsabilidades familiares como resultado de la presión social (Ballarín; García). Así, el entrevistado 23 indica: «Socialmente está más presionada, no todas, pero están socialmente más presionadas a hacer más cosas en casa, dedicarse más a la familia o a los hijos» (E.23. Hombre. Contratado doctor. Con hijos. Letras), o el participante 42 señala:

Esto que hablábamos antes de la necesidad de compatibilizar y todo eso. Yo creo que sí, que esa presión sigue estando más en las mujeres que en los hombres y eso a lo mejor lo explica, que a la mujer se le exige que no descuide su vida personal, mientras que en el hombre está como que más aceptado, que es normal, bueno pues que tiene que dedicar tiempo a su carrera y medrar y tal (E.42. Hombre. Cargo de gestión. Contratado doctor. Sin hijos. Letras).

En esta cita el entrevistado también reconoce que al contrario de lo que ocurre con las mujeres, en el caso de los hombres sí está socialmente aceptado que dediquen el tiempo que requieran a su carrera profesional. Por su parte, los discursos de las mujeres también dejan ver la presión social que sufren en relación al cuidado de los hijos. Estas exponen cómo personas muy cercanas a ellas continuamente le hacen comentarios criticando el tiempo que quitan a los hijos para dedicar al trabajo. En ningún caso los testimonios de los varones revelan que dicha presión social recaiga sobre ellos.

Por último, mientras parte del profesorado entrevistado considera a los hombres que colaboran en las tareas domésticas y de cuidado como una excepción; otra parte cree que la dinámica existente en relación al trabajo reproductivo está cambiando en las generaciones más jóvenes a favor de la corresponsabilidad, aunque reconocen que esta aún está lejos de ser una realidad:

Pero hoy en día sí que se están implicando mucho más. Yo lo veo aquí en la facultad, que hay muchos chicos que van a llevar a sus hijos al colegio y todas estas cuestiones, y bueno, las cosas van cambiando paulatinamente también. Se preocupan pero todavía usan la palabra yo ayudo. Lo cual quiere decir que alguien que es el que necesita ser ayudado es el que lleva la responsabilidad (E.9. Mujer. Catedrática de Universidad. Con hijos. Letras).

En síntesis, todo lo expuesto muestra, por un lado, que la conciliación de la vida personal y familiar con el desarrollo de la carrera profesional en la Universidad es muy complicada; y por otro, que especialmente lo es para quienes tienen cargas familiares, sean hijos $u$ otras personas mayores o enfermas a su cargo, y dentro de ellos, para las mujeres, que son quienes principalmente asumen estas funciones. Además, los testimonios también revelan que sigue existiendo presión social hacia el mantenimiento de esta situación 
aunque parte del profesorado se encuentra esperanzado por el cambio hacia la corresponsabilidad.

\subsection{La ausencia de corresponsabilidad como causa de la desigual promoción de las mujeres en la Universidad}

Con base en lo reflejado en el epígrafe anterior, algunos participantes consideran que la causa de la desigual posición de la mujer en la Universidad tiene que ver con su situación familiar (estos argumentos también aparecen en otras investigaciones, como la realizada por García de León y García de Cortázar):

Yo creo que en la universidad no, otra cosa es en la vida familiar. En mi departamento en este momento hay bastantes más mujeres que hombres, como sucede en casi todos los departamentos que hay en la zona de [dos áreas de conocimiento afines], y aquí yo no veo ninguna diferencia. Ahora, que la situación familiar condicione determinados aspectos de la cuestión laboral pues a lo mejor sí. Quiero decir: una mujer si tiene hijos y su situación es ella la que por lo que sea, la que se tiene que hacer cargo de los hijos siempre, pues a lo mejor eso le retrasa determinadas aspiraciones que pueda tener o hay una época ahí que pues escribe menos, que publica menos, que tal. Pero no es cuestión de la universidad, yo creo que es cuestión de la vida familiar que puede influirle ¿no? de cómo se monte la vida familiar (E.35. Hombre. Cargo de gestión. Titular de universidad. Con hijos. Letras).

Los hombres que defienden este tipo de argumentos no se cuestionan por qué la situación es tal, sino que ponen el peso del problema en la mujer o en el sistema social (García de León y García de Cortázar). Al contrario, la siguiente entrevistada incide en que esto sucede porque hay un problema de ausencia de corresponsabilidad en los hogares por parte de los varones, lo que obliga a las mujeres a realizar una doble jornada laboral:

Claro que influye en general la carrera profesional y el ser mujer, porque fundamentalmente, porque para poder conseguir el mismo currículum que un hombre, tú tienes que trabajar el doble de horas, pero no por nada, sino simplemente porque no hay corresponsabilidad en las tareas del hogar. No lo digo yo, lo dice la ONU. Con lo cual yo para publicar ocho artículos tengo que dedicar mucho más tiempo que un hombre que no tiene responsabilidades familiares, por decirlo de alguna manera. Porque él tiene todo el tiempo para dedicarlo a su carrera profesional. La mujer no, la mujer tiene que organizar su casa, tiene que organizar la comida, tiene que cuidar de los niños, tiene que cuidar de los ancianos (E.15. Mujer. Cargo de gestión. Contratada doctora. Sin hijos. Letras).

Cabe mencionar que aunque la entrevistada 15 indica que ella tiene que dedicar más tiempo al trabajo, entendido como la suma del trabajo productivo y reproductivo, que «un hombre que no tiene responsabilidades familiares», no 
se refiere a ello en sentido literal, sino que apela a que los hombres, tengan o no hijos $\mathrm{u}$ otras personas que dependan de su unidad familiar, no asumen las responsabilidades de cuidado sobre ellos, o al menos no en corresponsabilidad con sus parejas. Por su parte, la entrevistada 20 (que ocupa un cargo de gestión, es Titular de Universidad, no tiene hijos y trabaja en un área de Ciencias) reconoce que hay hombres que sí se ocupan de sus hijos, pero considera que no son mayoría. Ella, que no los tiene, cuenta la experiencia de sus compañeras madres, las cuales tienen que distribuir su tiempo de modo muy diferente a cómo lo suelen hacer los hombres que tienen hijos: una vez que acuestan a los niños, regresan al trabajo. Sus compañeros padres, por norma general, no necesitan hacerlo porque distribuyen el tiempo según sus necesidades, y no las de otras personas. La entrevistada se siente identificada con estas mujeres porque ella vivió una situación similar cuando cuidaba a su madre enferma. Durante unos años se vio obligada a finalizar su jornada laboral más temprano de lo que lo hacía habitualmente, y para compensar ese tiempo y continuar progresando en su carrera, volvía al trabajo de noche, cuando acostaba a su madre. De este modo, la entrevistada refleja que las mujeres no quieren dejar de progresar en la carrera académica, sin embargo, tratan de lograrlo desde situaciones de partida y condiciones muy diferentes a sus compañeros varones. Resulta llamativo que aparecen reivindicaciones de las mujeres hacia la falta de corresponsabilidad de sus compañeros de trabajo, debido a que si estos partiesen de una situación similar a la suya, competirían en igualdad de condiciones con ellos. Sin embargo, sus testimonios no reflejan que realicen esa misma reivindicación en sus propios hogares.

Algunas personas entrevistadas ejemplifican los casos de matrimonios en los que ambos miembros de la pareja son profesores de Universidad, como se observa en la siguiente cita extraída de la entrevista 3. En ninguno de ellos los modelos familiares apuntan hacia modelos de responsabilidad compartida. Al contrario, no solo evidencian la desigualdad en el hogar, sino también la desigual trayectoria profesional de unos y otras:

La mujer sigue estando pendiente de lo que ocurre en casa y de lo que ocurre a los hijos. Y yo jamás he visto... En, en mi facultad además se da el caso de que, bueno como pasa en muchas, que hay profesores que son maridos y mujeres, profesores y profesoras que son marido y mujer, y no, no, no. Siguen los patrones de pareja en el que la mujer sigue siendo la responsable de todo lo que ocurre en el ámbito familiar, siguen completamente vigentes [...] Todo sigue recayendo en su persona, en ellas, como mujeres, y no en sus maridos, que además, ¡suelen ser los catedráticos! (E.3. Mujer. Ayudante doctora. Sin hijos. Letras). 
El siguiente testimonio también habla de la experiencia de sus compañeras de trabajo, cuyas parejas son profesores de Universidad, y de cómo mientras ellos desarrollan su trabajo investigador de manera muy activa en todas las diferentes facetas del mismo, ellas se quedan más relegadas en él, por ejemplo, asistiendo a menos eventos de difusión científica que ellos. Esto se produce fundamentalmente como resultado de la asunción de responsabilidades en el seno de la familia. Al contrario, no aparecen testimonios que visibilicen la dinámica contraria:

Esto lo he hablado con más compañeras también profesoras Titulares, que a lo mejor, no es mi caso, o sea, mi marido no está en la universidad, pero tienen maridos en la Universidad, y es el marido al que se va a todos los congresos, cien mil en los años, y tú te quedas un poquito más, más limitada (E.6. Mujer. Titular de universidad. Con hijos. Ciencias).

Las dos siguientes citas provienen de profesoras cuyos maridos sí trabajan en la Universidad. La primera entrevistada señala que las responsabilidades familiares tienen un efecto diferente en la carrera de hombres y mujeres. Reconoce que ha habido ocasiones en las que le hubiera gustado implicarse en nuevos proyectos de investigación o haber ocupado otros cargos de gestión, pero que no ha podido hacerlo por cuestiones familiares. Al contrario, no señala este tipo de renuncias en su marido:

Sí, las mujeres vivimos peor en la universidad, eh, porque aún por muy igualitaria que mantenga las relaciones con la pareja, nunca es igual. Entonces tienes que asumir muchos aspectos de, de la vida doméstica, y de la familia, es decir, eh, preocuparte por los aspectos sentimentales, emocionales de los hijos. O sea ya no solo de intendencia, que normalmente es, sino también los aspectos emocionales. Entonces hay muchas veces, que pues te gustaría participar en un proyecto o cargarte con más responsabilidades en torno a investigación que no puedes, o, o, o gestión misma, eh, porque no puedes. [...] Y eso no significa que el compañero no te ayude ¿eh? Que en todos los casos posiblemente ayudan, pero, pero la carga, es, es, es de la mujer. Sí. (E.13. Mujer. Cargo de gestión. Titular de universidad. Con hijos. Letras).

Por su parte, la segunda entrevistada incide en que el trabajo reproductivo no afecta de igual modo a hombres que a mujeres. Señala que aunque en ocasiones las tareas a realizar finalmente se dividan, la responsabilidad de su organización no es compartida, sino que recae sobre ellas. Esta entrevistada también señala que los remordimientos de actuar de manera diferente a lo socialmente considerado también recaen sobre la mujer (Ballarín; García):

No, no, no, no. No afecta igual. No afecta igual, de ninguna manera. No afecta igual. [...]. Parece que es obligación nuestra, y aunque tu defiendas que no tiene porqué ser exclusiva tu obligación, y es verdad, y parte intentas 
compartirlo, el peso importante lo llevas tú. Aunque se comparta el tiempo. Y la responsabilidad también, la llevas tú. Y, y los remordimientos también los llevas tú. Todo. Aunque luego materialmente el tiempo se comparta, con ellos, ¿vale? Pero el peso, la organización, la distribución, sobre todo toda la organización. Si los niños necesitan tales vacunas, tales meses, hay que hacer no sé qué, hay que liberar ese día, que tengo que hacer no sé qué, tengo que pedirle a alguien que me cambie para que yo pueda ir a la vacuna o a la revisión, a no sé qué... Todo eso lo haces tú, y eso es claro. Claro que es: tensiones, estrés, es... el tener todo el mes ahí delante, para poder organizar, aunque luego sea para decir, es que este día tienes que ir tú, porque yo ya no he podido cambiar más cosas (E.10. Mujer. Catedrática de Universidad. Con hijos. Ciencias).

Cuando esta entrevistada compara su trayectoria profesional con la de su marido, las ve bastantes parejas, sin embargo, reconoce que al inicio de su relación ambos progresaban a la misma velocidad, y que esto cambió con el paso de los años y la asunción de responsabilidades familiares. Así, aunque ella también ha logrado ocupar una cátedra, lo consiguió unos años más tarde que su marido. En esta misma línea, el entrevistado 27, cuya mujer también es profesora de Universidad, reconoce que el efecto que la maternidad y las responsabilidades familiares en la carrera académica ha sido mayores en el caso de su mujer, aunque, en cierto modo, lo justifica: «Yo reconozco que ha tenido más influencia, probablemente, en la carrera académica de mi mujer, que en definitiva es quien ha tenido los niños ¿no?» (E.27. Hombre. Titular de Universidad. Con hijos. Letras). Este entrevistado reconoce que su mujer se doctoró, consiguió las primeras publicaciones y obtuvo las primeras plazas en la Universidad antes que él, logrando así una progresión académica mayor y más rápida hasta el momento en que se queda embarazada por primera vez. Desde entonces, la dinámica se modifica, y él comienza a aventajarla, hasta el punto de llevar años acreditado como catedrático de Universidad, al contrario que ella.

Por último, entre los testimonios de los varones, algunos reconocen haber llegado donde están gracias al apoyo de su mujer. Así lo hace el entrevistado 39, cuya mujer asumió el papel de ama de casa, ocupándose totalmente del trabajo doméstico y de cuidado de los hijos, liberándole a él de tales responsabilidades. Al contrario, ninguna mujer reconoce estar donde está porque su pareja apostara por su carrera laboral:

Si yo he conseguido, he llegado, estoy donde estoy, es, y no es una frase hecha que se suele hacer, es un altísimo porcentaje gracias a ella, porque a mí me ha liberado, me ha liberado de las cuestiones, en fin de lo que es la gestión de los asuntos más familiares, el cuidado de [Nombre de uno de sus hijos], [Nombre del otro de sus hijos], mis hijos. Hemos como marcado así un poco los territorios, pero sabiendo de antemano pues eso... Si no, yo estoy convencido que no 
podría haber hecho muchas de las cosas que he hecho, eso lo tengo clarísimo

(E.39. Hombre. Cargo de gestión. Titular de Universidad. Con hijos. Letras).

En síntesis, los participantes en la investigación consideran que una de las causas de la desigual posición de la mujer en la Universidad tiene que ver con su situación personal y familiar. Su mayor dedicación al ámbito reproductivo, en comparación con el hombre, estaría limitando su desarrollo y progresión en la carrera académica. En esta línea, Tobío (2012) considera que no será posible acabar con la desigualdad laboral por sexo hasta que no se logre una responsabilidad compartida en los hogares, ya que la ausencia de corresponsabilidad condiciona la energía de las mujeres (llegándoles a ocasionar problemas de salud, como ya se había señalado), limita su disponibilidad laboral y afecta a la toma de decisiones de estas en relación al trabajo.

\section{CONCLUSIONES Y REFLEXIONES}

Se ha producido una evolución del empleo del tiempo por sexo, lo que supone un reparto más equitativo del trabajo entre hombres y mujeres. Sin embargo, aún no hemos alcanzado el modelo de corresponsabilidad deseado. Los cambios sucedidos en la esfera pública, con el desarrollo económico y social del país y la masiva incorporación de la mujer al trabajo fuera del hogar, no se han trasladado a la esfera privada. Así, las relaciones existentes en este ámbito continúan constriñendo el tiempo social y generando diferencias significativas en el uso del tiempo en el trabajo remunerado y no remunerado por sexo. Esto genera una mayor carga global de trabajo para las mujeres y ocasiona importantes repercusiones para ellas tanto a nivel personal como laboral, limitando su acceso y promoción en el trabajo.

La conciliación de la vida familiar y el trabajo en la Academia es difícil para todos en la Universidad, por la dedicación de tiempo que esta carrera demanda, pero lo es especialmente para quienes tienen cargas familiares, y dentro de ellos, para las mujeres, ya que mayoritariamente son quienes se ocupan de su cuidado y atención. Además, los testimonios de las personas entrevistadas revelan que la mujer sigue estando socialmente más presionada para hacerse cargo del hogar y de estas tareas de cuidado. Mientras ellas soportan críticas sobre su doble dedicación al trabajo productivo y reproductivo, no parece existir una visión social negativa hacia que los hombres desatiendan el ámbito familiar. Por otra parte, y respecto al cambio en la tendencia en los modelos de reparto de responsabilidades, mientras unos participantes reconocen que se está produciendo dicho cambio, otros se muestran escépticos, considerando la existencia de hombres corresponsables como una excepción. Muchas de las personas entrevistadas consideran que la ausencia de corresponsabilidad en los 
hogares es una de las causas que contribuyen al mantenimiento de la desigual posición de la mujer en la Universidad. La carrera académica, por sus características, requiere de la dedicación de mucho tiempo privado al trabajo para poder progresar en ella. Sin embargo, el tiempo es, como se ha señalado, un recurso desigualmente distribuido. Así, la mayor dedicación al trabajo reproductivo por parte de las mujeres genera, por un lado, que dispongan de menos tiempo para dedicar al trabajo productivo y, por otra, que para progresar en la carrera académica requieran hacer malabares entre unas responsabilidades y otras, llegando incluso a tener que trabajar de noche, una vez que acuestan a los niños. De este modo, las mujeres no quieren dejar de progresar en la carrera académica pero tratan de lograrlo desde situaciones de partida y condiciones muy diferentes a los hombres. Los testimonios de las personas cuya pareja también es académico/a son muy reveladores. Muestran cómo el progreso laboral de ambos se fue produciendo de manera equiparada, o incluso que el ascenso de la mujer era más rápido en los inicios, hasta que aumentan las responsabilidades familiares. Desde entonces, los hombres comienzan a aventajar a sus mujeres en la progresión en la carrera académica.

Estos resultados plantean retos sobre el modelo social y sobre el propio diseño de la carrera académica. Respecto al primero, resulta llamativo, por un lado, el mantenimiento de modelos no corresponsables en parejas donde ambos miembros trabajan, especialmente porque ambos tienen un alto nivel de formación y desarrollan un trabajo muy especializado; por otro, que la ausencia de corresponsabilidad también esté presente en las parejas más jóvenes; y por último, que no haya un mayor reclamo por parte de estas mujeres hacia la corresponsabilidad en sus hogares. Respecto al modelo de carrera académica, estamos ante un tipo de carrera en la que no se puede parar, que requiere de la dedicación excesiva de tiempo para poder progresar en ella, reduciendo el tiempo que debería dedicarse a la familia, el ocio o el descanso. Así, es más fácil alcanzar las principales posiciones para aquellos que menos responsabilidades tienen en el hogar o menos se hacen cargo de ellas, debido a la disponibilidad de emplear su tiempo libre al trabajo. Siendo así, debemos plantearnos si este es el modelo de desarrollo de la ciencia que queremos, a costa de las personas. Teniendo presente todo ello, se requieren aún muchos y profundos cambios sociales para asegurar, por un lado, la responsabilidad compartida entre hombres y mujeres en los hogares, y por otro, las mismas oportunidades laborales para unos y otras. Necesitamos modelos sociales en los que se dé por hecho que todas las personas cuidan (y para lograrlo, políticas sociales que trabajen en esa línea), y con ellos, que se espere y exija a todas la misma dedicación y responsabilidad. Y de manera paralela, necesitamos modelos de carrera que 
tengan esto en cuenta, y que ajusten las demandas que hacen a sus trabajadores para que puedan desarrollarse profesionalmente sin necesidad de desatender el ámbito familiar o de elegir entre ambas esferas. En caso contrario, no solo se mantendrá la desigualdad sino que se está desaprovechando parte del talento de la mitad de la población en su intento de hacer compatible trabajo y familia. Y considero, como afirma Monteserín, que desaprovechar ese talento tiene graves consecuencias para la sociedad, tanto a nivel económico como demográfico y social.

\section{REFERENCIAS BIBLIOGRÁFICAS}

Acker, Sandra. «Gender, leadership and change in faculties of education in three countries». Leadership, gender and culture in education. Ed. John Collard y Cecilia Reynolds. Maindenhead, UK: Open University Press, 2005, 103-117.

Álvaro, Mariano. «Diferencias, en el uso del tiempo, entre hombres y mujeres y otros colectivos.» Revista Española de Investigaciones Sociológicas 74 (1996a): 291-326.

Álvaro, Mariano. Los usos del tiempo como indicadores de la discriminación entre géneros. Madrid: Instituto de la Mujer, 1996.

Anguita, Rocío, Ana Isabel Alario y Teresa Ortiz. Las mujeres en la Universidad de Valladolid. Valladolid: Secretariado de Publicaciones e Intercambio Editorial, Universidad de Valladolid, 2003.

Ballarín, Pilar. La educación de las mujeres en la España contemporánea (siglos XIXXX). Madrid: Síntesis, 2001.

Barberá, Ester y Amparo Ramos. «Liderazgo y discriminación de género.» Revista de la Federación Española de Asociaciones de Psicología 57 (2004): 147-160.

Beck, Ulrich y Elisabeth Beck-Gernsheim. La individualización: el individualismo institucionalizado y sus consecuencias sociales y políticas. Barcelona: Paidós, 2003.

Carrasco, María José. «Participación y poder de las mujeres en las organizaciones educativas.» XXI Revista de Educación 6 (2004): 75-87.

Conde-Ruiz, J. Ignacio e Ignacio Marra. Gender gaps in the Spanish labor market. FEDEA, 2016.

Domínguez, Marta. «La división del trabajo doméstico en las parejas españolas. Un análisis del tiempo.» Revista Internacional de Sociología 70 (2012): 153-179.

Domínguez, Marta. «Parenthood and domestic division of labour in Spain, 20022010.» Revista Española de Investigaciones Sociológicas 149 (2015): 45-62.

Donoso, Trinidad, Pilar Figuera y María Luisa Rodríguez. «Barreras de género en el desarrollo profesional de la mujer universitaria.» Revista de Educación 355 (2011): 187-212.

Durán, María Ángeles. La jornada interminable. Barcelona: Icaria, 1986. 
Durán, María Ángeles. Tiempo de vida y tiempo de trabajo. Bilbao: Fundación BBVA, 2010.

García de León, María Antonia y María Luisa García de Cortázar. Las académicas: Profesorado universitario y género. Madrid: Instituto de la Mujer, 2001.

García, Teresa. «Carrera profesional de las maestras y matrimonio.» Aula Abierta 86 (2005): 87-119.

González, María José y Teresa Jurado. Padres y madres corresponsables. Una utopía real. Madrid: Catarata, 2015.

Gutek, Barbara y Laurie Larwood. «Women's careers are important and different.» Women's career development. Ed. Barbara Gutek y Laurie Larwood. Newbury Park, California: Sage Publications, 1987, 7-14.

Hochschild, Arlie. The second shift: Working families and the revolution at home. New York: Wiking, 1989.

Instituto de la Juventud. Sondeo de opinión. $3 .^{\circ}$ Encuesta. Jóvenes e igualdad de género. Madrid: INJUVE, 2008.

Instituto de la Mujer. Usos del tiempo, estereotipos, valores y actitudes. Madrid: Instituto de la Mujer, 2007.

Instituto de la Mujer. «Estadísticas. Usos del tiempo I», 2013. <http://inmujer.es/ MujerCifras/Conciliacion/UsosdelTiempo.htm>, consultado el 15-12-2016.

Lozares, Carlos, Pedro López y Joel Martí. «La relación entre los tiempos y las actividades del trabajo productivo y reproductivo.» Trabajo 13 (2004): 165-186.

Martínez-Pérez, María Dolores y Amparo Osca. «El éxito profesional desde una perspectiva de género: propuesta de un modelo.» Revista de Psicología General y Aplicada 51.2 (2004): 193-208.

Ministerio de Educación, Cultura y Deporte. 2017. «Estadística de Personal de Universidades (EPU). Curso 2015-2016», 2017. (http://www.mecd.gob.es/ servicios-al-ciudadano-mecd/estadisticas/educacion/universitaria/estadisticas/ personal-universitario.html), consultado el 10-06-2017.

Monteserín, Mariví. «La mujer en la empresa ¿hay una mano invisible?» Mujeres en la alta dirección. La carrera profesional de las mujeres en la empresa, la administración y la Universidad. Ed. Capitolina Díaz y Elena Carantoña. Madrid: Instituto de la Mujer, 2008, 116-119.

Murillo, Soledad. El mito en la vida privada: de la entrega al tiempo propio. Madrid: S. XXI, 1996.

Prieto, Carlos, Ramón Ramos y Javier Callejo. Nuevos tiempos del trabajo: Entre la flexibilidad competitiva de las empresas y las relaciones de género. Madrid: Centro de Investigaciones Sociológicas, 2008.

Ramos, Ramón. Cronos dividido: uso del tiempo y desigualdad entre mujeres y hombres en España. Madrid: Instituto de la Mujer, 1990.

Reskin, Barbara. "Sex differentiation and the social organization of science.» Sociological Inquiry 48.3-4 (1978): 6-37. 
Tharenou, Phyllis. «Is there a link between family structures and women's and men's managerial career advancement?» Journal of Organizational Behavior 20.6 (1999): 837-863.

Tobío, Constanza. «La familia y el empleo: prácticas y discursos de las mujeres.» Cuestiones Actuales de Sociología del Género. Ed. Rita Radl. Madrid: Centro de Investigaciones Sociológicas, 2001, 129-148.

Tobío, Constanza. 2012. «Cuidado e identidad de género. De las madres que trabajan a los hombres que cuidan.» Revista Internacional de Sociología 70.2 (2012): 399-422.

Tomàs-Folch, Marina y Cristina Guillamón. «Las barreras y los obstáculos en el acceso de las profesoras universitarias a los cargos de gestión académica.» Revista de Educación 350 (2009): 253-275.

Varella, Reyes. «Las políticas de igualdad en el ámbito del trabajo: una propuesta de construcción teórica desde el género.» Cuestiones actuales de Sociología del Género. Ed. Rita Radl. Madrid: Centro de Investigaciones Sociológicas, 2001, 105-125. 